Service social

\title{
Service social auprès des gais et intervention de groupe
}

\section{Pierre Berthelot}

Volume 37, numéro 1-2, 1988

Par-delà les barrières des sexes

URI : https://id.erudit.org/iderudit/706392ar

DOI : https://doi.org/10.7202/706392ar

Aller au sommaire du numéro

Éditeur(s)

École de service social de l'Université Laval

ISSN

1708-1734 (numérique)

Découvrir la revue

Citer cet article

Berthelot, P. (1988). Service social auprès des gais et intervention de groupe. Service social, 37(1-2), 197-213. https://doi.org/10.7202/706392ar

\section{Résumé de l'article}

Cet article a été élaboré à partir d'une expérience réalisée à Québec, en 1986, dans laquelle l'auteur a tenté de rejoindre des hommes homosexuels éprouvant des difficultés à s'accepter, pour leur proposer de rompre leur isolement par une expérience de groupe. Quelques appuis théoriques sont identifiés pour analyser le problème et préciser l'intervention. La jonction théorie-expérience permet de retenir les éléments les plus importants pour l'intervention de groupe auprès de personnes homosexuelles dans une optique de développement personnel. 
BeRTHELOT, Pierre, responsable du réseau de support psychosocial, Équipe de prévention et de dépistage, infection à V.I.H. et Sida, Région de Québec.

\title{
Service social auprès des gais * et intervention de groupe
}

\section{Pierre Berthelot}

\begin{abstract}
"On n'a peut-être pas assez remarqué que le problème de la liberté sensuelle sous toutes ses formes est en grande partie un problème de liberté d'expression. Il semble bien que, de génération en génération, les tendances et les actes varient peu; ce qui change au contraire est autour d'eux l'étendue de la zone de silence ou l'épaisseur des couches de mensonge. " 1
\end{abstract}

Marguerite Yourcenar

Cette réflexion, écrite en 1963, représente bien l'attitude des sociétés nord-américaines devant le phénomène de l'homosexualité. Au Québec, les principales institutions au pouvoir, jadis inflexibles envers les personnes homosexuelles, sont devenues tolérantes : au plan légal, l'orientatin sexuelle a été incluse, en 1977, dans la Charte des droits et libertés de la personne comme motif pour lequel on ne peut exercer de discrimination; au plan médical, suivi de près par le champ psychosocial, on ne considère plus, officiellement, le désir homosexuel comme pathologique ; au plan religieux, l'Église catholique, qui hait le péché tout en aimant le pécheur, accepte les homosexuels mais désapprouve leur homosexualité. Que notre société s'accommode de l'existence de personnes qui pensent et agissent selon une orientation affective et sexuelle différente de la norme, voilà un premier pas: des homosexuels se sont rendu visibles, affirmant leur désir comme une capacité plutôt que comme une limite. En ce sens, les mouvements d'activistes gais, développés aux États-Unis dans les années 1970, ont 
facilité cette libération en incitant les gais à briser leur isolement et en remettant en question les valeurs sociales relatives aux rôles de l'homme et de la femme. Bel exemple de l'effet que peuvent produire la mobilisation et le regroupement d'individus qui désirent prendre leur place dans la société et se débarrasser des stéréotypes sclérosants! À l'instar de leurs sympathisantes féministes, ils ont remis en cause la valeur suprême du mâle dominant. Un journal anglais de libération homosexuelle déclarait, en 1970 :

"Nous constatons que l'oppression subie par les personnes homosexuelles fait partie intégrante des structures de la société. Les femmes et les personnes homosexuelles sont deux groupes d'individus victimes de ce phénomène culturel qu'est le sexisme et qui se manifeste par la suprématie mâle et le chauvinisme hétérosexuel. »2

En 1989, des milliers d'hommes et de femmes ne vivent plus selon les schèmes traditionnels, et des milliers d'autres essaient de s'en défaire. C'est dans une telle optique qu'à Québec, en 1986, nous avons tenté de rejoindre des hommes homosexuels éprouvant des difficultés à s'accepter, pour leur proposer de rompre leur isolement par une expérience de groupe engageante.

L'expérience s'est avérée positive. Avant sa réalisation, nous avions identifié quelques appuis théoriques pour définir, analyser le problème et préciser les objectifs à poursuivre pour le résoudre. La jonction théorie-résultats de l'expérience nous a permis de retenir les éléments les plus importants pour l'intervention de groupe auprès de personnes homosexuelles dans une optique de développement personnel.

\section{Les dimensions de la question}

\section{Définition et cadre d'analyse}

Nous empruntons au psychiatre Judd Marmor sa définition de I'homosexualité.

"L'homosexuel est quelqu'un qui, dans sa vie adulte, est motivé par une attraction érotique définie et préférentielle pour les membres de son propre sexe. Cette personne s'engage habituellement (mais pas nécessairement) dans des relations sexuelles avec un partenaire du même sexe. ${ }^{3}$

Il est judicieux d'abord de retenir qu'il s'agit d'une attraction "définie et préférentielle", excluant ainsi toute manifestation homosexuelle circonstancielle ouvrant la possibilité que la personne vive 
également des attractions hétérosexuelles. On souligne avantageusement aussi que "l'homophile» est d'abord animé par un désir qu'il peut actualiser ou non dans son comportement.

Puisque aucune définition n'est tout à fait complète, on nous permettra quelques commentaires. D'abord, on situe l'homosexualité à l'âge adulte, sans tenir compte des manifestations homosexuelles de l'enfance et de l'adolescence qui, pourtant, font partie du développement "psychosexuel» de l'être humain. Une autre limite de cette définition est qu'elle ne fait aucune mention du contexte social dans lequel l'individu exprime ou non sa pulsion sexuelle, alors que ce contexte est fort important dans la mesure où il exerce des pressions sur la personne homosexuelle.

Pour définir l'homosexualité, nous omettons intentionnellement l'aspect étiologique au même titre que la recherche des cures. Traiter ces questions équivaudrait à considérer le comportement homosexuel comme pathologique; nous endossons plutôt la position de Tripp ${ }^{4}$ qui le définit, au même titre que l'hétérosexualité, comme une pulsion sexuelle ne pouvant pas s'articuler sur des apprentissages négatifs. Selon lui, on érotise les attributs de son propre sexe à cause de l'intérêt intrinsèque qu'il représente, et non à défaut de pouvoir développer une orientation hétérosexuelle. Une telle perspective permet de situer le phénomène de l'homosexualité au niveau de l'interaction entre l'homosexuel et la société, interaction problématique parce que l'individu concerné doit se développer et s'épanouir dans une société " hétérosexiste " qui rend son orientation sexuelle marginale, anormale, voire pathologique, et qui, par certaines de ses institutions (médicales, religieuses, légales) ne le reconnaît pas ou l'opprime pour l'amener à se conformer aux modèles de pensée et de comportement qu'elle juge désirables. Ces institutions n'accordent aucun reflet véritablement positif et surtout aucun support au style de vie homosexuel. Au cours des deux dernières décennies, on a officiellement "dépathologisé » et décriminalisé l'homosexualité, mais on est loin de l'avoir intégrée dans nos mœurs : les attitudes sociales devant le phénomène le démontrent bien.

\section{Attitudes sociales}

L'oppression par les attitudes sociales a une influence considérable sur le développement du gai. Elles s'expriment dans le vécu quotidien par l'opinion publique, les modèles de pensée et d'action véhiculés par l'éducation et les communications, les gestes et les paroles adressés directement ou non à un individu et qui contribuent à la construction 
de son identité. Pour recevoir des autres l'amour et l'estime qui permettent de se sentir soi-même aimable et désirable, c'est-à-dire s'estimer, une personne tend d'abord à se conformer aux modèles. En cas d'échec, l'équilibre est menacé.

Par ailleurs, c'est un fait généralement admis que l'homosexualité est mal vue dans notre société et que les idées véhiculées à son égard sont plus souvent négatives que positives. Tantôt elle est perçue comme une maladie, tantôt comme un vice. On prête souvent aux homosexuels des caractéristiques ne reposant sur aucun fondement. Ces préjugés et stéréotypes sont d'ailleurs très souvent intégrés par les gais eux-mêmes, qui on i bien du mal à s'en défaire. On rencontre le plus fréquemment trois types d'attitudes sociales devant l'homosexualité : la persécution, la discrimination et la tolérance.

La persécution est une oppression violente infligée aux individus ou aux groupes homosexuels qui agissent ouvertement ou qu'on identifie comme tels par certaines caractéristiques extérieures correspondant aux stéréotypes qu'on leur accole habituellement. Ces personnes sont sujettes à des agressions verbales et physiques de la part des gens normaux: l'agression de rue par de petits voyous, le harcèlement policier, l'utilisation vicieuse de l'homosexualité par les médias de troisième ordre en l'associant à des histoires de meurtre ou de viol, les railleries quotidiennes, les farces de mauvais goût.

La discrimination touche surtout les personnes connues comme homosexuelles, et ce, même si elles n'agissent pas ouvertement comme telles, et même dans des situations où leur vie affective et sexuelle n'est pas du tout en cause. C'est le cas du domaine de l'emploi, plusieurs estiment que la discrimination qui s'y manifeste est l'une des principales raisons qui font craindre aux gais de s'identifier comme tels. Hormis certains secteurs plus ouverts qui valorisent la différence, la carrière d'un individu peut être compromise s'il est connu comme gai ou soupçonné de l'être. C'est le cas des militaires, des juristes, des politiciens, des religieux, des enseignants et des éducateurs. Cette discrimination s'exerce également au moment de recourir à certains services publics.

La tolérance, c'est-à-dire le fait de ne rien interdire ni exiger alors qu'on le pourrait, est l'attitude à laquelle les homosexuels sont le plus exposés de nos jours. ${ }^{5}$ C'est l'attitude des gens bien, l'attitude de bien des gens. On la retrouve chez les personnes libérales qui, intellectuellement, ne pourraient accepter d'être hostiles aux gais, mais qui, au fond d'elles-mêmes, ne reconnaissent pas ce style de vie comme valable. Leur tolérance est loin d'être une acceptation profonde et véritable : il faut la considérer comme une générosité accordée par un supérieur à un subalterne. C'est le cas de religieux et de plusieurs "professionnels de l'acceptation inconditionnelle de l'autre ». Soulèverait-on légèrement 
le masque libéral de ces personnes bien intentionnées qu'on trouverait, intacte, l'empreinte de toutes les idées négatives sur l'homosexualité laissées par l'éducation. Si ces gens ont tort, ce n'est pas tant d'avoir des préjugés que de ne pas les reconnaître. En les camouflant, ils se privent de l'enrichissement qu'apportent l'écoute véritable et l'ouverture aux points de vue différents.

\section{"L'auto-oppression "}

C'est dans ce contexte d'inconfort social devant l'homosexualité que certaines personnes découvrent leur préférence pour des personnes du même sexe et tentent de l'intégrer dans leur vie. Conséquemment, prendre conscience de ses désirs homosexuels conduit l'individu à une crise personnelle intense causée par la remise en question de l'image de soi construite à partir des modèles sociaux " hétérosexistes ". Plus encore, une personne se voit animée par des désirs qu'elle avait appris à condamner bien avant d'en soupçonner l'existence chez elle. Elle cherchera alors à lutter contre ses attirances, à les invalider, à les refouler et surtout à les cacher. L'anxiété et la peur d'être découverte sont grandes, car cette révélation implique la possibilité d'une baisse d'estime ou d'un rejet de la part des personnes qui lui sont significatives.

Aussi longtemps qu'une personne garde son orientation sexuelle imperceptible, elle reste sujette aux attaques involontaires (raillerie, farce de mauvais goût, commentaire négatif) qui finissent par compromettre sérieusement l'estime de soi. Le temps passe, les désirs homosexuels persistent et, si elle continue à vivre dans un milieu défavorable à l'homosexualité, cette personne se convainc qu'elle est inadéquate et anormale. Le fait de voiler son secret par des mensonges contribue à renforcer l'image négative qu'elle a d'elle-même. L'insistance à demeurer caché génère et alimente "l'auto-oppression", soit : garder le silence, se refuser à toute relation affective et sexuelle satisfaisante, éviter les modèles homosexuels positifs, s'abstenir de rechercher du support, s'interdire le droit de vivre dans un milieu adapté à sa personnalité originale. Être son propre oppresseur, c'est mener une double vie, dans la crainte constante du rejet, et feindre l'hétérosexualité dans la vie quotidienne. À plus long terme, cette "auto-oppression" risque de s'actualiser par toutes sortes de situations d'échec dans diverses zones de l'existence et par des comportements " autopunitifs " pouvant même aboutir au suicide, ultime conséquence d'une interaction problématique entre un individu homosexuel et une société qui a une vision étriquée de la sexualité humaine. 


\section{L'identité gaie positive}

Fort heureusement, une grande proportion des personnes homosexuelles réussissent à développer et à épanouir leur orientation affective et sexuelle en dépit de toutes ces contraintes. Leur démarche n'est toutefois pas facile et, pour certains, s'étale sur plusieurs années.

À un stade avancé de son cheminement, l'individu a intégré l'homosexualité à l'ensemble de sa personnalité : c'est ce que nous appelons l'identité gaie positive. Avoir intégré cette identité, c'est avoir assumé sa composante homosexuelle et se définir non seulement à partir de son orientation sexuelle qui n'est qu'une composante de la personnalité, mais plutôt de façon globale. Il n'existe pas de portrait type d'une telle personne, puisque l'identité n'est pas un état statique mais implique une intégration continuelle d'expériences nouvelles. Il y a toutefois des indicateurs. D'abord, une personne ayant assimilé positivement son homosexualité est capable de s'engager de façon préférentielle dans des relations affectives, sexuelles et intellectuelles avec des personnes du même sexe. Elle est capable de témoigner ou non de son orientation sexuelle en toute liberté et la vit comme une source de satisfaction et d'épanouissement; en toutes circonstances, elle est de plus en plus authentique et sait choisir les relations qui lui renvoient une image positive d'elle-même. On peut dire enfin qu'elle s'affirme et s'estime davantage. Pour en arriver là, plusieurs auront dû vivre un cheminement personnel dont les composantes se retrouvent dans le modèle d'intervention que nous abordons maintenant.

\section{L'intervention}

\section{Une expérimentation préalable}

Notre modèle d'intervention est issu de la synthèse des éléments les plus significatifs de la littérature portant sur le vécu psychosocial des personnes homosexuelles et des résultats d'une intervention de groupe réalisée à Québec, en 1986, auprès d'hommes homosexuels.

L'intervention utilisée se guide sur le modèle développemental de Tropp ${ }^{6}$ et prend la forme d'un groupe de croissance personnelle, composé d'hommes âgés de 18 à 35 ans et désireux d'être aidés dans leur démarche d'acceptation d'eux-mêmes. Bien que faisant face à des difficultés d'acceptation et d'affirmation de leur orientation "psychosexuelle ", ils présentent un niveau d'équilibre personnel satisfaisant. Il s'agit bien d'un groupe de croissance et non de thérapie. Le 
principal objectif de l'intervention, répartie en seize rencontres hebdomadaires de trois heures, est d'accompagner chaque participant dans sa démarche d'intégration d'une identité gaie positive.

Pour façonner l'image du groupe, le modèle de Tropp propose un contrat à partir des objectifs individuels reliés, dans la mesure du possible, aux objectifs communs. Le groupe est un lieu, un milieu de prise de conscience des perceptions de chacun; il est essentiellement autodéterminé et chaque participant est tout à fait libre sur ce qu'il veut donner ou recevoir du groupe et sur les changements personnels qu'il veut opérer. Quant aux activités, centrées sur "l'ici et maintenant", elles doivent encourager les relations égalitaires entre les membres et l'engagement personnel de chacun, et s'adressent au groupe en tant qu'entité à travers laquelle chacun peut travailler à sa propre croissance. Choisies en fonction des besoins et des ressources des membres, ce sont des activités de prise de conscience des liens existant entre le corps et les émotions, des discussions de thèmes ou de documents audiovisuels, des activités d'expression par l'écriture, le dessin, les mises en situation.

Dans cette expérience, le travailleur social est à la fois participant, facilitateur, catalyseur. Il s'implique en partageant, comme les autres du groupe, émotions et expériences, tout en gardant assez d'objectivité pour remplir son rôle. Il est surtout mobilisateur de l'énergie et de la créativité des participants en vue de l'atteinte des objectifs.

Les séances ont lieu le soir, de $19 \mathrm{~h} 30$ à $22 \mathrm{~h} 30$, et comprennent six étapes : mise en train, détente, mise en situation, pause, retour sur la mise en situation, retour sur la séance.

Une mise en train d'environ quinze minutes est faite d'exercices physiques (sauts, étirements) qui visent à donner de l'énergie aux participants, tout en leur permettant de libérer une partie des tensions de la journée. Chacun est libre de participer et on respecte le rythme de chacun. En général, tous s'adonnent aux exercices et en apprécient les bienfaits. Les participants ont ensuite vingt minutes de détente dirigée où ils sont amenés à se concentrer sur leur corps et leurs émotions. Cette première demi-heure les dispose aux exercices suivants et les aide à entrer en contact plus intime avec eux-mêmes.

Suit la mise en situation, noyau de la séance, qui développe le thème de la rencontre. L'exercice, axé sur le vécu d'un événement plutôt que sur la discussion, fait vivre des expériences sensibles sur lesquelles on pourra réfléchir ensuite ; il exige une implication personnelle, qui s'accroîtra au fil de l'évolution du groupe. Les thèmes, dont nous donnons la liste plus bas, ne sont pas tous spécifiques au vécu homosexuel, plusieurs concernent tout être humain. Ces thèmes universels prennent une coloration particulière du fait qu'on les aborde en 
étroite relation avec le vécu gai et dans un groupe composé uniquement de personnes homosexuelles du même sexe :

- le premier contact d'une relation,

- les interdits sociaux et culturels,

- les valeurs,

- la vie amoureuse,

- la révélation de son homosexualité dans sa famille,

- la communication non verbale,

- l'affirmation de soi,

- les critères d'attrait physique,

- l'estime de soi,

- l'authenticité dans une relation de couple,

- la perception des autres,

- les fantasmes sexuels.

Tous sont ensuite invités à écrire ou à représenter visuellement dans un album ce qu'ils ont retenu.

Voici un exemple de mise en situation. Lors d'une séance dont le thème est Témoigner de son homosexualité dans sa famille, un participant qui projette inviter bientôt sa mère pour lui parler de son orientation sexuelle, joue son propre rôle. Récemment, la mère a appris, par l'une de ses filles, l'homosexualité de son fils. La projection de cette rencontre est très anxiogène pour le participant. Dans la mise en situation, le travailleur social joue le rôle de la mère qui adopte une attitude culpabilisante. Cet exercice rejoint tous les participants pour qui la révélation est une démarche projetée ou réalisée.

Après l'exercice, c'est la pause, moment fort de la séance où l'on discute le plus du vécu gai en société. L'hétérogénéité du groupe a ici beaucoup d'impact : ceux qui vivent un degré d'acceptation personnelle plus élevé peuvent témoigner aux autres des aspects positifs de l'orientation homosexuelle, tout en comprenant très bien leurs difficultés, puisqu'ils ont franchi les mêmes étapes et livré des batailles similaires pour reconquérir l'estime d'eux-mêmes. Ils peuvent également les appuyer dans leur démarche en leur indiquant certaines ressources où trouver du support (bars, services de santé, services psychosociaux, groupes de discussion, lectures). Quant à ceux qui en sont aux premières étapes vers l'identité positive, ils trouvent une occasion de dépasser leur insécurité pour découvrir progressivement des modèles d'identification positifs, qui ne sont pas visibles dans leur environnement social immédiat, et qui démontrent qu'à travers sa différence un homosexuel peut s'épanouir au même titre qu'une personne hétérosexuelle. La pause permet aussi aux participants d'échanger des informations, de suggérer des lectures, de partager des points de vue. Àl'animateur, elle 
donne de précieux indicateurs des préoccupations des membres et lui fournit des idées de thèmes pour les séances ultérieures.

Après la pause, le groupe complète le travail sur les émotions en échangeant sur la mise en situation. Librement, chaque personne peut livrer ses impressions sur ce qu'elle vient de vivre. Les verbalisations sont reliées aux émotions ressenties, à la qualité des contacts avec les autres, aux aspects de la situation vécus plus ou moins facilement. Il est de règle que, pendant cette période, aucun jugement de valeur ne doit être porté sur les messages transmis au groupe. Quand un participant intervient, c'est pour demander des précisions à l'émetteur ou pour lui donner du support. À une seconde étape, les participants recherchent les liens possibles entre la façon dont ils ont vécu la mise en situation et celle dont ils vivent les situations quotidiennes semblables. Par exemple, dans un jeu de rôle ayant trait à un conflit dans le couple, l'un des protagonistes avait tu une légitime agressivité compte tenu du contexte particulier de l'exercice; lors du retour, il fait un lien direct avec plusieurs situations de sa vie de couple où il a réagi pareillement.

Ici, l'animateur ne tente pas d'amener le groupe à des conclusions générales mais oriente plutôt les interactions pour que chacun en tire les conclusions qu'il estime applicables à sa vie. On respectera entièrement celui qui ne ferait aucun lien entre son attitude dans l'exercice et celle qu'il adopte dans une situation quotidienne similaire.

À la fin de chaque rencontre, on réserve une vingtaine de minutes pour que les participants s'expriment sur le vécu de la séance. C'est le moment de dire ce qui a plu ou non dans les activités, de manifester son degré de satisfaction, de suggérer des thèmes pour les soirées futures, de proposer des modifications à la formule et d'énoncer des considérations relatives à la vie "socio-affective" du groupe.

Cette expérience d'intervention a fait l'objet, dans un essai antérieur ${ }^{7}$, d'une évaluation systématique portant sur ses effets et sur la satisfaction des participants. Notons brièvement que les participants sont très satisfaits de l'expérience. En tenant compte des limites d'une évaluation, on peut raisonnablement avancer que le groupe aide les participants à s'affirmer davantage, qu'il leur permet de trouver un lieu de partage sur leur vécu et les expériences reliées ou non à l'homosexualité et qu'il leur fait connaître les ressources pertinentes à une meilleure intégration sociale. L'intervention semble contribuer à modifier les perceptions et les attitudes des participants dans le sens d'une acceptation plus grande de leur orientation homosexuelle.

Ces conclusions viennent confirmer la position de Don Clark ${ }^{8}$ qui a inspiré l'ensemble de l'intervention et, par conséquent, les principaux concepts du guide d'intervention. 


\section{Un modèle de pratique}

Selon Murisier-Lalonde, ${ }^{9}$ un modèle de pratique est : « un ensemble structuré de pensées et d'actions, de pensées liées à des actions, ensemble qui se présente comme un univers de réponses, de choix cohérents aux questions de l'agir professionnel ».

Les composantes d'un modèle de pratique sont: les postulats de base, les principes d'action, la stratégie globale, les objectifs généraux et spécifiques, les moyens, les rôles et le pouvoir des acteurs.

\section{Les postulats de base}

Assises du modèle de pratique, ils lui donnent sa cohérence. Un postulat est un principe premier appuyé sur des travaux de recherche mais non démontré scientifiquement, et posé au départ pour établir une démonstration. Pour notre modèle, nous proposons trois postulats.

Premier postulat: L'homosexualité est une orientation sexuelle viable, compatible avec l'épanouissement d'un être humain, au même titre que l'hétérosexualité.

Certains auteurs, dont Benoît, ${ }^{10}$ affirment que l'homosexualité est une orientation sexuelle normale. Nous exprimons ici notre réticence à utiliser le terme normal, car nous ne voulons pas poursuivre le débat relatif à la normalité de l'homosexualité, des siècles d'histoire nous ayant démontré qu'il s'agit là d'une aventure hasardeuse. D'ailleurs, ce qui nous importe est l'épanouissement de la personne et non sa "normalité».

Deuxième postulat : Considérant l'hétérosexualité seule orientation sexuelle valable, la société confère à la personne homosexuelle un statut de marginal.

À cet effet, on a vu quelles sont les pressions sociales auxquelles une personne homosexuelle est confrontée et dont elle doit s'affranchir pour épanouir son orientation sexuelle et affective.

Troisième postulat: Les pressions sociales qui s'exercent sur la personne homosexuelle peuvent entraver sérieusement le développement de l'estime de soi.

\section{Les principes d'action}

De ces postulats découlent des principes qui guideront l'action et le programme d'intervention. Le premier principe reconnaît que l'intervention s'adresse à une personne adéquate qui a des difficultés de 
relation dans la société. Le second croit que l'amélioration des conditions de la vie d'un homosexuel doit nécessairement passer par une intervention sur la personne elle-même tout autant que sur les structures sociales.

\section{La stratégie globale}

Découlant de la conception théorique du problème, la stratégie globale constitue le cadre général de l'action en vue d'un objectif spécifique. Pour notre modèle, la stratégie idéale devrait faire porter l'intervention, d'une part sur la personne elle-même et, d'autre part sur les groupes humains dont elle fait partie. Pour l'individu, elle devrait viser une plus grande estime personnelle en tant qu'homosexuel et, pour la société, elle devrait tendre à la modification des perceptions envers la sexualité et l'homosexualité.

La stratégie concerne donc d'abord l'homosexuel : elle tente d'améliorer sa perception de lui-même pour devenir plus fort face aux pressions sociales et plus habile à faire évoluer les perceptions qu'ont de l'homosexualité les personnes qui l'entourent. Il s'agit de développement personnel et la stratégie doit tenir compte de quatre nécessités énoncées par Clark ${ }^{11}$.

La déprogrammation : Toute personne qui découvre son orientation homosexuelle doit reconnaître les valeurs " hétérosexistes " qu'on lui a inculquées et voir si elles correspondent à sa personnalité. Elle doit ainsi se déprogrammer de toutes les conceptions non adaptées à ses besoins qu'elle a fait siennes et qui la placent en situation de conflit "intrapersonnel ".

Contact avec son corps et ses émotions: À cause des préjugés négatifs qu'ils ont intégrés, et pour être conformes à ce qu'ils croient qu'on attend d'eux, certains gais passent par une phase de négation d'eux-mêmes, de refoulement de leurs désirs homosexuels et, par conséquent, de l'ensemble de leurs émotions. Dans sa tentative de se mériter l'approbation sociale, si un individu refoule ses émotions, il lui faut aussi, pour que le refoulement soit efficient, anesthésier une partie de ses sensations corporelles. Pour se développer et s'épanouir, il lui faut reprendre contact avec ses émotions et avec son corps, qui en est le véhicule. C'est ainsi que le développement d'un contact plus intime avec son corps et avec ses émotions est une seule et même tâche.

Recherche de support : $\mathrm{Si}$, dans les années qui ont suivi la découverte de son orientation, l'homosexuel a été isolé et privé de références positives, il doit fréquenter d'autres gais qui, ayant cheminé positivement, sont susceptibles de le guider et de l'appuyer dans sa démarche. 
Sortir: Sortir, c'est affirmer son identité gaie. Il n'est pas dans le répertoire des habiletés socialement acquises que d'informer un proche de son orientation affective et sexuelle différente, quoique cette démarche soit nécessaire au rétablissement de l'estime de soi. Cacher délibérément son homosexualité, c'est renforcer, à ses propres yeux, l'idée qu'elle soit honteuse. Et sortir face aux autres s'avère une expérience souvent fortifiante lorsque les gens à qui on a choisi de se révéler le reçoivent comme une marque de confiance. Les relations n'en deviennent que plus solides parce que la personne y est authentique.

\section{Les objectifs}

Un objectif se définit comme un ensemble d'effets projetés par un agent de changement sur un environnement donné.

L'objectif général du modèle est en lien direct avec la problématique. Il sera d'aider l'homosexuel à cheminer dans le développement d'une identité gaie positive. On dit d'une telle personne qu'elle a intégré l'homosexualité à l'ensemble de sa personnalité: elle a assumé sa composante homosexuelle et est capable d'avoir un concept global d'elle-même qui ne s'élabore pas seulement à partir de sa préférence sexuelle. Aussi, elle est capable de s'engager dans des relations affectives, sexuelles et intellectuelles avec des personnes du même sexe, de façon préférentielle. Elle vit son orientation sexuelle comme une source de satisfaction et d'épanouissement personnel.

L'objectif général du modèle s'articule en des objectifs spécifiques en rapport avec la problématique, les postulats de base et les principes d'action.

- Faire émerger chez l'individu la prise de conscience des obstacles extérieurs (préjugés, informations erronées, discrimination) qui gênent le développement harmonieux de sa vie affective et sexuelle.

- L'informer adéquatement de façon à faire contrepartie aux données biaisées qu'il peut avoir reçues.

- Développer chez l'individu un contact plus intime avec ses sensations corporelles et ses émotions, et en faciliter l'expression.

- Briser l'isolement.

- Faire connaître les ressources accessibles aux gais dans le milieu où il vit.

- Fournir des modèles positifs de vécu homosexuel.

- Lui permettre de faire l'expérience de l'affirmation de son 
homosexualité dans un environnement qui lui renvoie une image positive de lui-même.

- Encourager l'expression de l'imagination et de la créativité.

\section{Les moyens}

Les moyens que nous privilégions pour atteindre ces objectifs proviennent de différentes composantes de l'intervention appelée groupe de croissance personnelle. II s'agit d'une intervention auprès d'un groupe constitué de personnes du même sexe et d'orientation homosexuelle. Il nous paraît essentiel que les membres soient du même sexe puisqu'il s'agit, pour eux, de faire l'expérience d'affirmer ouvertement des sentiments et des désirs pour des personnes du même sexe. II ne serait pas contre-indiqué de regrouper des personnes homosexuelles des deux sexes, mais cela devrait se faire plus tard, dans un programme ultérieur qui pourrait traiter d'une façon plus générale du vécu homosexuel en société.

Le groupe de croissance personnelle s'inspire du modèle du développement de l'individu où le client est perçu comme sain et capable de réalisations personnelles et de progrès continuels. Ce modèle met de l'avant l'établissement d'un contrat à partir des objectifs individuels en lien avec les objectifs du groupe. Le groupe est un lieu de prise de conscience des perceptions de chacun. Il est essentiellement autodéterminé et chaque participant, libre de changer, est autonome quant à ce qu'il veut donner et recevoir du groupe.

À partir de l'évaluation de notre expérience, voici ce qu'on devra retenir pour le recrutement et la sélection des participants, le rôle du travailleur social et les thèmes à aborder.

Recrutement et sélection : Le groupe idéal se situe entre huit et douze personnes, pour permettre des interactions nombreuses ainsi que le développement d'un sentiment d'intimité. Les participants retenus doivent reconnaître leur orientation homosexuelle, mais ceci n'implique pas qu'ils doivent avoir solutionné tous les problèmes reliés à cette orientation. Il serait trop anxiogène pour quelqu'un qui doute de son orientation véritable de se joindre à un groupe d'individus se reconnaissant homosexuels. Pour ces personnes en situation de crise ou d'ambivalence, une relation d'aide individuelle s'avère beaucoup plus indiquée.

Puisqu'il s'agit d'un groupe de croissance et non de thérapie, il faut éviter de sélectionner des gens qui vivent des difficultés autres que celles reliées à l'homosexualité. On doit regrouper des individus fonctinnels dans les diverses sphères de leur vie. C'est pourquoi il faut 
orienter ailleurs les personnes alcooliques, toxicomanes ou psychiatrisées, ou encore prévoir des groupes spécifiques de thérapie pour des homosexuels vivant l'une ou l'autre de ces difficultés.

Dans la phase de préparation du programme, on doit prévoir une durée totale de seize à vingt semaines pour l'intervention, de sorte que le groupe ait le temps nécessaire pour vivre toutes les phases de son développement.

Rôle du travailleur social : D'abord, il est préférable que l'intervenant soit lui-même homosexuel et qu'il ait cheminé positivement dans P'intégration de son orientation afin de présenter un modèle positif de référence. Cependant, un intervenant hétérosexuel qui connaît bien le vécu personnel et social des gais, et qui est bien situé lui-même par rapport à ses propres tendances homosexuelles, peut s'avérer tout aussi efficace. II n'est pas nécessaire à la réussite de l'intervention que le professionnel vive une situation similaire à celle de ses clients. Il s'agit par contre d'être réellement ouvert et d'avoir fait le point sur soimême. Un intervenant défensif par rapport à sa composante homosexuelle mais qui se dit ouvert aux gais, risque d'émettre inconsciemment des messages négatifs à travers ses attitudes non verbales.

Les principaux rôles du travailleur social sont d'être attentif au cheminement du groupe et à l'écoute de ses membres, de respecter le rythme des individus et du groupe, de stimuler la participation et les interactions, de préserver le groupe de la stagnation, de permettre à chacun d'y prendre sa place, de présenter des exercices originaux facilitant l'expression et l'intimité de chacun avec ses propres réactions corporelles et émotives. L'intervenant a un rôle à jouer dans l'établissement du climat de confiance qui s'installera progressivement, dans la mesure où certaines règles de base sont acceptées par tous, la principale étant que les membres du groupe ne portent aucun jugement de valeur sur ce qui est exprimé pendant les séances. Lorsqu'un participant prend la parole, les autres sont invités à réagir en posant des questions qui amèneront l'émetteur à explorer plus en profondeur le message émis ou en lui donnant du support. Mais en aucun temps les jugements ne seront tolérés. De cette manière, chacun acquiert la certitude que ses messages seront reçus et acceptés tels quels. Les gens n'ont pas à s'approuver ou à se désapprouver entre eux, mais plutôt à reconnaître et à accepter leurs différences. Ainsi, l'hétérogénéité peut devenir une force pour le groupe et une source d'enrichissement pour chacun.

En ce qui a trait à la façon d'aborder les divers thèmes du programme, le travailleur social doit présenter des mises en situation amenant les membres à vivre des expériences concrètes qui les mobilisent entièrement plutôt que de laisser libre cours aux discussions purement intellectuelles qui ne s'adressent qu'à la raison et qui n'impliquent ni le corps ni les 
émotions. Les discussions rationnelles ne sont pas néfastes, elles peuvent même être aidantes, mais elles ne nous semblent pas les plus appropriées compte tenu des objectifs poursuivis.

Thèmes: Nous croyons que l'intervenant doit d'abord consulter les participants sur les sujets qu'ils aimeraient aborder puisque le groupe est essentiellement autodéterminé. II n'est pas obligatoire que tous les thèmes traités soient directement reliés à l'homosexualité, bien que cela nous semble préférable. Dans le cas où un thème plus général est traité, on doit le relier le plus possible au vécu homosexuel.

\section{Vers une plus grande acceptation sociale de l'homosexualité}

Aider les personnes homosexuelles à acquérir une identité gaie positive ne constitue qu'une réponse partielle à la résolution de la problématique reliée à l'homosexualité si aucun autre effort ne tente de modifier les attitudes sociales envers elles. Bien que cet aspect n'ait pas constitué le centre de notre expérience, il n'en demeure pas moins tout aussi important.

Weinberg et Williams ${ }^{12}$ notent que la relation des gais avec le monde hétérosexuel est susceptible d'influencer leur équilibre psychologique. Moins un individu craindra d'être découvert, moins il anticipera de rejet et de discrimination, et plus il sera sujet à avoir un fonctionnement psychologique satisfaisant. C'est pourquoi il est important d'intervenir sur l'interaction négative entre les groupes homosexuels et l'ensemble de la société de façon à modifier positivement cette interaction.

Des progrès du côté des attitudes sociales pourraient se traduire par une diminution des craintes irrationnelles entretenues dans la population à propos de l'homosexualité et par une nouvelle façon de la percevoir, non plus comme une déviation ou une inversion, mais comme une composante de la sexualité humaine. C'est pourquoi les communautés gaies (groupes de support clinique ou de santé, activités socio-culturelles, établissements commerciaux spécialisés) doivent être supportées et renforcées, car en plus de constituer une étape saine dans le cheminement individuel vers l'identité gaie positive, elles peuvent être des agents de transformation positive des préjugés et des mentalités de la société qui confère au style de vie homosexuel un statut déprécié. De telles communautés doivent tendre au maintien de relations égalitaires avec le reste de la société pour que s'installe une véritable acceptation entre gais et non-gais. Un grand travail d'éducation reste à faire. II faut intervenir sur les conceptions de la sexualité en général, car notre 
société est encore très marquée par les tabous et c'est une des principales raisons qui fait que l'homosexualité est mal vue par le plus grand nombre. Cette réalité est d'autant plus vraie en cette fin des années 1980, au cœur d'une épidémie de sida, ce mal compliqué, mortel, transmis sexuellement à $97 \%$, qui, en Amérique du Nord, a jusqu'à présent atteint les homosexuels dans près de $80 \%$ des cas connus.

Comment pourrait-on faire évoluer, dans un tel contexte, les perceptions sociales de l'homosexualité ? par des campagnes d'éducation et d'information dans la population ? en établissant des liens de solidarité avec d'autres groupes marginalisés pour exercer des pressions sur les institutions au pouvoir? en éduquant les étudiants aux valeurs du libre choix sexuel et du respect des différences? en mettant sur pied, dans les cégeps et les universités, des cours sur les problématiques reliées à la sexualité ?... Possiblement ! Par le biais de témoignages positifs et par la mobilisation des gais eux-mêmes, fiers de leur préférence?... Inévitablement !

\section{Notes et références}

* L'usage exclusif du masculin n'a pour but que d'alléger le texte puisque l'objet de cet article concerne également des femmes et des hommes.

1 Marguerite Yourcenar, Alexis ou le traité du vain combat, Paris, Gallimard, 1971 : $12-13$.

2 Jim Carrigan, Bob Connell, et John Lee, "Toward a new sociology of masculinity ", dans; Harry BrooD (éd.), The Making of Masculinities, London, Allen \& Unwin, 1987: 63-102.

3 Judd MARMOr (éd.), Sexual Inversion, New York, Basic Books, 1965, 358p.

4 C.-A. Tripp, The Homosexual Matrix, New York, McGraw Hill, 1975, p. 12.

5 Dennis Altman, Homosexuel(le) oppression et libération, Paris, Éditions Fayard, 1976, p. 58.

6 Emmanuel Tropp, "A developmental theory", Robert-W. ROBERT et Helen NORTHERN (éds), Theories of Social Work with Groups, New York, Columbia University Press, 1976, 401p.

7 Pierre Berthelot, Service social des groupes auprès des gais : vers un modèle de pratique, Sainte-Foy, Université Laval, Ecole de service social, février 1988 : 48-69.

8 Don Clark, Loving Someone Gay, Berkeley (Cal.), Celestial Arts, 1977, p. 34.

9 Mary-Lise MURISIER-LALONDE, Modèle de pratique du service social en milieu scolaire, Montréal, Université de Montréal, École de service social, avril 1976, p. 18. 
10 Jacques BENOÎT, Étude préliminaire à l'implantation de services psychosociaux pour la communauté homosexuelle de la région 03, Sainte-Foy, Université Laval, École de psychologie, 1980.

11 Don Clark, op. cit.

12 Martin-S. Weinberg, et Collin-J. Wiluiams, Male homosexuals, Their Problems and Adaptation, New York, Oxford University Press, 1974, pp. 123-125. 